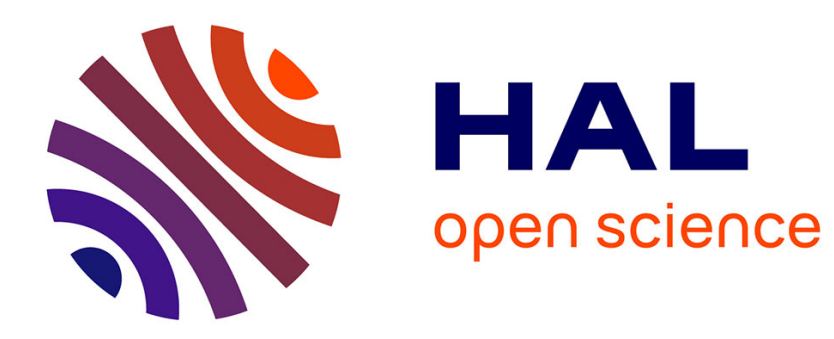

\title{
Propriétés contractiles des fibres musculaires et performance physique chez le cheval \\ Eric Barrey
}

\section{To cite this version:}

Eric Barrey. Propriétés contractiles des fibres musculaires et performance physique chez le cheval. Productions Animales, 1994, 7 (1), pp.41-53. hal-00896075

\section{HAL Id: hal-00896075 \\ https://hal.science/hal-00896075}

Submitted on 1 Jan 1994

HAL is a multi-disciplinary open access archive for the deposit and dissemination of scientific research documents, whether they are published or not. The documents may come from teaching and research institutions in France or abroad, or from public or private research centers.
L'archive ouverte pluridisciplinaire HAL, est destinée au dépôt et à la diffusion de documents scientifiques de niveau recherche, publiés ou non, émanant des établissements d'enseignement et de recherche français ou étrangers, des laboratoires publics ou privés. 
INRA Prod. Anim., 1994, 7 (1), 41 - 53.

\section{E. BARREY}

INRA Station de Génétique quantitative 78352 Jouy-en-Josas Cedex
Propriétés

contractiles des

fibres musculaires

et performance

physique chez le cheval

On dit d'un cheval qu'il a "du sang" lorsqu'il a une constitution athlétique, les veines à fleur de peau et un tempérament vif. Mais, sur le plan physiologique, que recouvre exactement cette notion empirique connue des hommes de chevaux depuis des temps immémoriaux? Quelle relation y-a-t-il entre la vivacité du tempérament, la puissance physique d'un cheval et ses caractéristiques physiologiques? Les connaissances acquises en physiologie musculaire permettent maintenant de répondre en partie à ces questions. Les qualités contractiles et métaboliques du tissu musculaire déterminent, en effet, la puissance et la durée des contractions que le muscle pourra fournir au cours d'un exercice.

La vitesse et la force de contraction développée par un muscle dépendent à la fois de la quantité de fibres actives et de leurs propriétés contractiles et métaboliques. Sur le plan de la vitesse de contraction, il existe chez l'homme, et plus généralement chez les mammifères, deux principales catégories de fibres musculaires : les unes à contractions lentes, les autres à contractions rapides. La typologie musculaire consiste en l'étude des caractéristiques contractiles et métaboliques des différents types de fibres qui composent les muscles. Les fibres musculaires de type I ont une vitesse de contraction lente, et sont capables de se contracter successivement un

\section{Résumé}

Cet article présente les connaissances acquises sur la physiologie des fibres musculaires en relation avec l'exercice physique chez le cheval-athlète. La vitesse et la force de contraction développée par un muscle dépendent à la fois de la quantité de fibres actives et de leurs propriétés contractiles. Le muscle étant le moteur de l'athlète, de nombreuses études de physiologie de l'exercice ont porté sur la typologie musculaire afin d'en connaître le déterminisme et les potentialités adaptatives en réponse à un travail d'entraînement. Les méthodes d'investigation, les caractéristiques fonctionnelles, les facteurs de variation et les relations avec la performance physique sont présentés et discutés dans le cas du cheval pour envisager une application à la sélection. grand nombre de fois mais avec une force modérée, tandis que les fibres de types IIA et IIB, à contractions rapides, développent des forces plus élevées mais pendant peu de temps. Pour assurer leur fonctionnement, les fibres musculaires sont dotées d'un métabolisme énergétique à prédominance aérobie ou anaérobie en fonction de leur type : les lentes (I) sont aérobies, les rapides sont soit aéroanaérobies (IIA), soit anaérobies (IIB). De par leurs caractéristiques contractiles et métaboliques, les fibres de type I favorisent l'exercice d'endurance (de faible intensité pendant plusieurs heures), tandis que les fibres de type II permettent d'effectuer un exercice de puissance (d'intensité élevée pendant quelques dizaines de secondes). Ainsi, en fonction de la composition en fibres lentes et rapides des différents muscles locomoteurs d'un athlète tel que le cheval, celui-ci présentera une meilleure aptitude à soutenir un effort d'endurance ou de puissance. Le muscle étant le moteur de tout athlète, de nombreux travaux de physiologie de l'exercice ont porté sur la typologie musculaire afin d'en déterminer les propriétés, le déterminisme et les potentialités adaptatives. L'objet de cet article est de passer en revue les connaissances acquises sur la typologie musculaire chez le cheval en relation avec son aptitude à l'exercice physique. 


\section{1 / Exploration de la typologie musculaire chez le cheval}

\section{1 / Prélèvement par biopsie}

Les études de physiologie musculaire chez l'homme et les autres espèces sportives ont nécessité la mise au point d'une technique de prélèvement peu traumatique (Lindholm et Piehl 1974, Bergstrom 1975, Snow et Guy 1976). L'athlète ne doit bien évidemment subir aucune détérioration musculaire et doit pouvoir reprendre son activité physique rapidement.

Figure 1. Prélèvement d'un échantillon de 10 à $30 \mathrm{mg}$ sur le fessier moyen à l'aide d'une aiquille à biopsie de faible diamètre $(2 \mathrm{~mm})$.

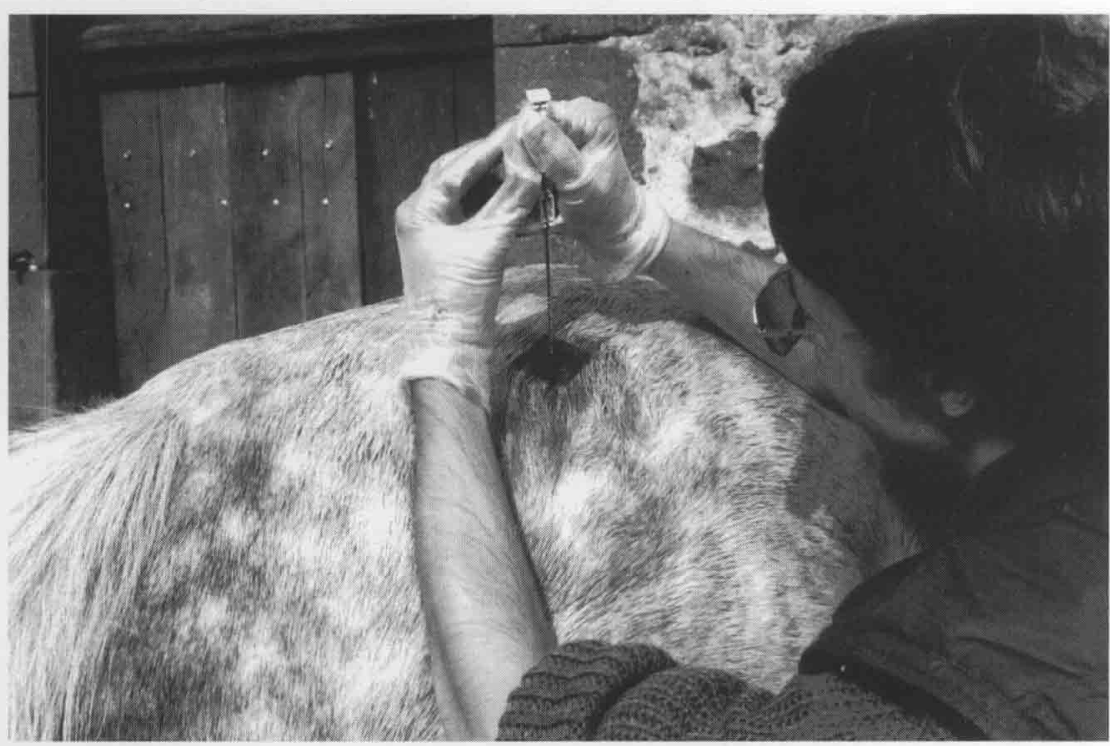

(E. Barrey INRA, Jouy)

Figure 2. Coupe transversale d'un muscle de cheval préparé par la technique de coloration histoenzymatique révélant l'activité ATPasique des différents types de myosine (d'après Gottlieb-Vedi 1988). Les fibres les plus foncées sont du type lente aérobie (I) ; les fibres les plus claires sont du type rapides aéro-anaérobies (IIA); les fibres de teinte intermédiaire sont du type rapide anaérobie (IIB).

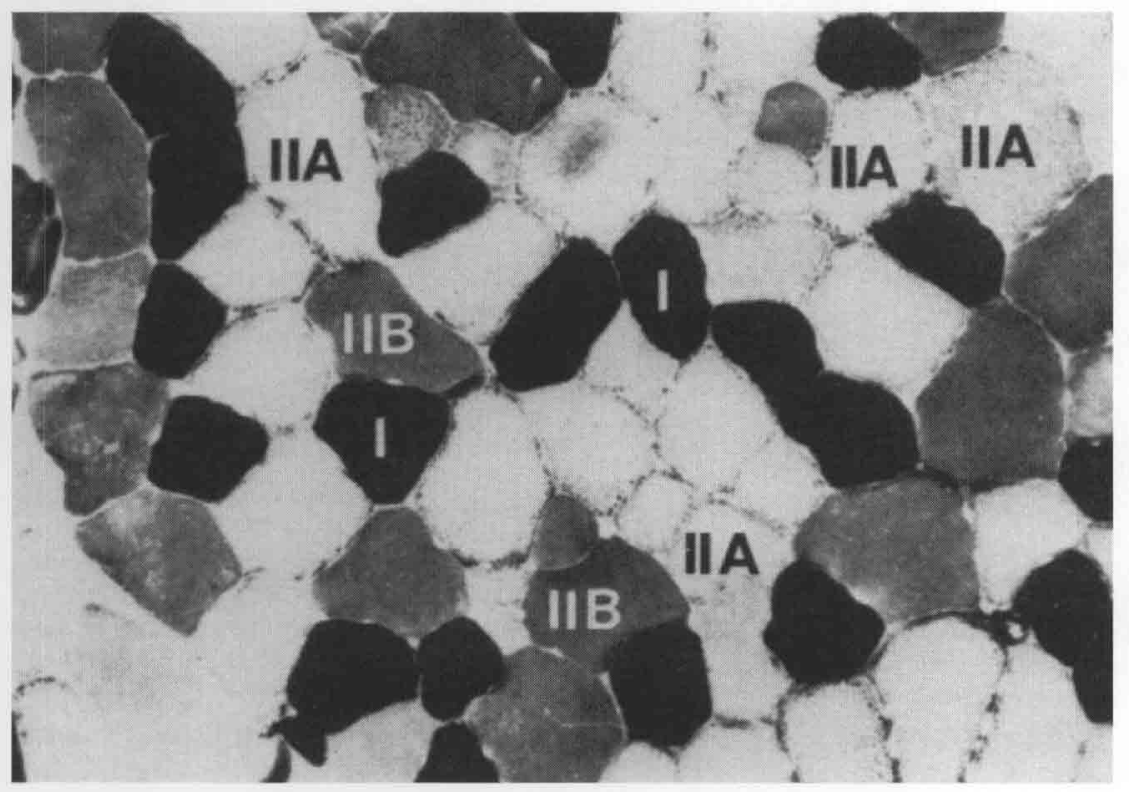

(M. Gottlieb-Vedi, Swedish University, Uppsala)
L'emploi d'aiguille à biopsie de petit diamètre $(2$ à $5 \mathrm{~mm}$ ) permet de prélever en toute sécurité des échantillons de muscle de 10 à 100 $\mathrm{mg}$ à une profondeur de 5 à $8 \mathrm{~cm}$ (figure 1 ). Sur le cheval, on effectue d'abord un nettoyage antiseptique sur la zone préalablement tondue; puis, 10 minutes après une anesthésie locale sous-cutanée, on pratique une petite incision cutanée jusqu'à l'aponévrose musculaire. Ceci permet de passer l'aiguille à biopsie directement dans le muscle sans avoir à transpercer la peau qui est très sensible chez le cheval. La biopsie ainsi réalisée n'est pas douloureuse, la cicatrisation est nette et rapide et le cheval peut retravailler sans risque une heure après. Plusieurs aiguilles sont disponibles mais toutes fonctionnent sur le même principe. L'aiguille de Bergstrom (1975) de $5 \mathrm{~mm}$ de diamètre est utilisée chez l'athlète humain et le cheval pour prélever des échantillons de taille suffisante pour l'histologie (environ $100 \mathrm{mg}$ ). Dans nos travaux sur des chevaux de sport ou de course, nous préférons utiliser des aiguilles de plus faible diamètre $(2 \mathrm{~mm})$ guère plus traumatisantes qu'une simple aiguille à injection intramusculaire (Barrey et al 1994, Valette et al 1994). Cette méthode de biopsie requiert cependant d'autres techniques de dosage que l'histologie car la masse de tissu prélevée n'est que de 10 à $30 \mathrm{mg}$.

Le muscle prélevé est immédiatement placé dans un tube pour être congelé dans l'azote liquide et conservé jusqu'au moment de l'analyse. Pour effectuer des coupes histologiques, il faut talquer ou humecter d'isopenthane l'échantillon afin de faciliter la réalisation des coupes histologiques.

\section{2 / Méthodes d'analyse}

Les analyses du tissu musculaire caractérisent le type métabolique (aérobie, anaérobie ou mixte) ou le type contractile (lent ou rapide) des fibres musculaires par différentes techniques. On en distingue trois catégories : l'histologie, l'électrophorèse et l'immunoenzymologie. Les plupart des travaux chez le cheval et l'athlète humain ont eu recours à une technique histologique : l'histoenzymologie. Elle a l'avantage de donner de nombreuses caractéristiques sur les fibres musculaires mais sa mise en œuvre est très longue (Lindholm et Piehl 1974, Snow et Guy 1980). C'est cette méthode qui a été à l'origine de la classification des fibres selon les types I, IIA et IIB à laquelle nous nous référerons dans cet article.

\section{a / Méthodes de typage histoenzymatique}

Après dissection de la biopsie musculaire, des coupes transversales à l'axe des fibres musculaires sont préparées au microtome pour être ensuite colorées spécifiquement. Les différences d'activité enzymatique des ATPases de la myosine sont révélées par une double incubation des coupes dans des solutions tampon acide ( $\mathrm{pH} 4,3$ puis 4,6 ), puis alcaline $(10,3)$. Au microscope, les fibres lentes (I) apparaissent alors en foncé, les fibres rapides IIÂ en clair et les fibres rapides IIB en gris (figure 2). Le dénombrement de 200 à 500 fibres sur chacune 
des coupes histologiques conduit au calcul des proportions numériques des trois types de fibre. La surface relative des fibres musculaires peut être mesurée par des procédés informatisés d'analyse d'images. D'autres colorations peuvent être utilisées pour mesurer la densité des capillaires et des mitochondries.

\section{b / Méthodes de typage par électrophorèse}

Les différences de poids moléculaire et de charges électriques des chaînes lourdes de la myosine lente et rapide permettent de les séparer par la technique d'électrophorèse sur gel de polyacrylamide. Si l'on désire une simple mise en évidence ou un dosage semiquantitatif de chaque type de myosine, la méthode de séparation par électrophorèse est utilisable (Billeter et al 1987, Hermanson et al 1991). La lecture des bandes par un densitomètre fournit une mesure quantitative de la composition en fibres des trois types. La séparation des fibres lentes et rapides est facile à réaliser, les fibres I étant celles qui migrent le plus lentement. Par contre, la séparation des fibres rapides IIA et IIB est plus délicate. L'identification des bandes peut se faire à l'aide de deux références en myosine lente et rapide : le muscle de la joue (masséter) qui ne contient que de la myosine lente et un muscle cutané (cutaneus tronci) qui ne contient que des fibres rapides (Picard et al 1994). On peut également utiliser des anticorps monoclonaux anti-myosine lente et rapide pour identifier les bandes sur le gel, il s'agit alors de la technique dite "d'immunoblotting".

\section{c / Méthodes de typage immunologique}

Les chaînes lourdes de la myosine lente et rapide ont des propriétés antigéniques distinctes qui ont permis de préparer des anticorps monoclonaux anti-myosine lente et antimyosine rapide (Winkelman et al 1983, Léger et al 1985, Danieli Betto et al 1986) ou encore anti-alphatubuline (Horak et al 1991). L'emploi de ces anticorps en association avec différents procédés de marquage permet d'effectuer soit des dosages quantitatifs (Immunoblotting et Enzyme Linked Immunoabsorbent Assay ou ELISA), soit des colorations immunohistologiques. L'emploi d'anticorps monoclonaux dirigés contre la myosine lente ou rapide présente l'intérêt d'une grande sélectivité de dosage ou de marquage histologique.

La technique ELISA de dosage de la myosine lente utilisée chez les bovins, est aussi applicable depuis peu chez le cheval (Barrey et al 1994, Picard et al 1994, Valette et al 1994). Elle permet d'effectuer une analyse quantitative de la proportion de myosine lente (MHC 1) contenue dans une biopsie musculaire. Par rapport aux méthodes histologiques, cette technique est plus rapide et permet de traiter par série un grand nombre d'échantillons. Cette méthode de dosage permet l'emploi de petites aiguilles à biopsie peu traumatisantes. En effet, l'intégralité de l'échantillon musculaire prélevé peut être dosée puisque, contrairement à l'histologie, l'orientation des fibres n'intervient pas. L'analyse par technique immunoenzymatique porte ainsi sur une quantité pondérale de muscle bien supérieure à celle d'une coupe histologique (400 à 600 fois plus dans le cas d'un prélèvement avec une aiguille à biopsie de $2 \mathrm{~mm}$ ). C'est semble-t-il une méthode d'avenir pour les analyses musculaires de routine en physiologie de l'exercice.

Les techniques d'immunohistologie permettent d'identifier sur des coupes sériées les fibres lentes et les fibres rapides (Sinha et Rose 1992). La distinction entre types IIA et IIB est cependant encore impossible chez le cheval car il n'existe pas d'anticorps anti IIA ou anti IIB comme chez le rat (Danieli Betto et al 1986). Quelques rares fibres ont une teinte intermédiaire entre lentes et rapides, correspondant au fait qu'elles contiennent les deux types de myosine MHC 1 et MHC 2A. On les appelle les fibres IIC.

\section{3 / Classification des fibres musculaires}

Plusieurs nomenclatures de typologie musculaire ont été proposées selon les espèces et les méthodes d'analyse mises en cuvre. La notion de muscle à fibres rouges ou blanches n'est pas applicable chez le cheval car toutes les fibres contiennent de la myoglobine qui donne une teinte rouge plus ou moins foncée. La classification des fibres basée sur la révélation de l'activité enzymatique de la myosine ATPase a été largement adoptée en physiologie de l'exercice depuis les premier travaux de Brooke et Kaiser (1970) qui mettaient en évidence les différents types de fibres musculaires. En histoenzymologie, on distingue donc chez le cheval 3 principaux types de fibres : un type à contractions lentes (I) et deux types à contractions rapides (IIA et IIB). Selon le mode d'incubation des coupes histologiques, on peut également trouver des fibres intermédiaires IIC (entre I et IIA) en faible proportion (moins de $1 \%$ en général) chez l'adulte comme chez le poulain (Roneus et Lindholm 1991, Roneus 1993). Le tableau 1 récapitule les principales caractéristiques de chaque type de fibre. Le type contractile de la fibre selon la coloration A'TPasique est étroitement lié à ses propriétés métaboliques : aérobie (I), anaérobie (IIB) ou aéro-anaérobie (IIA, IIC).

Les analyses électrophorétiques et immunologiques étant plus spécifiques des caractéristiques moléculaires de la myosine, elles amènent maintenant à compléter cette classification. L'étude de la composition en myosine à l'intérieur de chaque fibre des muscles chez le rat a montré qu'il pouvait exister des chaînes lourdes de la myosine différentes dans une même fibre (Pette et al 1977, Danieli Betto et al 1986). Ainsi, les fibres lentes I sont constituées de 57 à $100 \%$ de MHC 1 et le complément de MHC 2A. Les fibres rapides IIA contiennent 69 à $100 \%$ de MHC $2 \mathrm{~A}$ et le complément en MHC 1 et MHC 2B. Les fibres rapides IIB contiennent un mélange de $\mathrm{MHC}$ $2 \mathrm{~B}$ et $\mathrm{MHC} 2 \mathrm{~A}$.

\section{Les fibres musculaires se distinguent essentiellement par leur vitesse de contraction et leur métabolisme.}


Tableau 1. Caractéristiques des 3 principaux types de fibres musculaires chez le cheval. La richesse en capillaires, myoglobine et mitochondries est schématisée par les ",,++++++ "qui indiquent les quantités relatives entre les différents types de fibres.

\begin{tabular}{|l|c|c|c|}
\hline & Fibre I & Fibre IIA & Fibre IIB \\
\hline Contraction & lente $100-200 \mathrm{~ms}$ & rapide $40-88 \mathrm{~ms}$ & rapide $40-88 \mathrm{~ms}$ \\
Unité motrice & peu de fibres & nb moyen de fibres & beaucoup de fibres \\
Vitesse de conduction & + & ++ & +++ \\
de l'axone & plusieurs heures & plusieurs minutes & moins d'une minute \\
Fatigabilité & +++ acides gras & ++ & + \\
Métabolisme aérobie & + & +++ glycogène & ++ glycogène \\
Métabolisme anaérobie & +++ & ++ & + \\
Capillaires, myoglobine, & + & ++ & +++ \\
mitochondries & & & + \\
Surface des fibres & & &
\end{tabular}

\section{2 / Caractéristiques fonctionnelles des fibres musculaires}

\section{1 / Propriétés structurales contractiles et métaboliques}

La figure 3 schématise la structure moléculaire de la myosine qui est constituée de 2 chaînes lourdes et de 4 chaînes légères. Les différences de qualités contractiles résultent de la combinaison des éléments de base de la myosine : lente ou rapide. Les chaînes légères interagissent avec les filaments d'actine pour former les points d'ancrage nécessaires au raccourcissement de la fibre. Les têtes de la myosine constituées de l'association des chaînes lourdes et légères sont le siège de l'activité ATPasique qui permet la contraction. Pour caractériser le type lent ou rapide, ce sont les chaînes lourdes qui sont dosées spécifiquement par un anticorps (Léger et al 1985, Danieli Betto et al 1986, Picard et al 1994).

Les quatre types de fibres se distinguent par leurs propriétés contractiles et métaboliques. Chaque muscle comporte un mélange de ces différents types de fibres dans des proportions variables selon sa fonction.

Les fibres lentes (I) ont une vitesse de contraction 3 fois plus faible que celle des fibres rapides et un temps de relaxation 5 fois plus long (Gollnick 1982). Ces fibres I développent une force maximale inférieure à celle des rapides mais la répétition des contractions peut durer plus longtemps sans apparition de phénomène de fatigue (baisse de la force de contraction). Le métabolisme énergétique est exclusivement aérobie et il consomme plus particulièrement des acides gras par l'intermédiaire de la Béta-oxydation. Ces caractéristiques métaboliques s'accompagnent d'une forte concentration de myoglobine, d'une densité élevée de mitochondries et de la présence de nombreux capillaires en périphérie de la fibre. Tous ces éléments contribuent à l'apport et à l'utilisation de l'oxygène nécessaire au métabolisme aérobie de cette fibre spécialisée pour les contractions successives de longue durée. Il existe des muscles très riches en fibres lentes
Figure 3. Schéma de la structure moléculaire de la myosine (d'après Gollnick 1982).

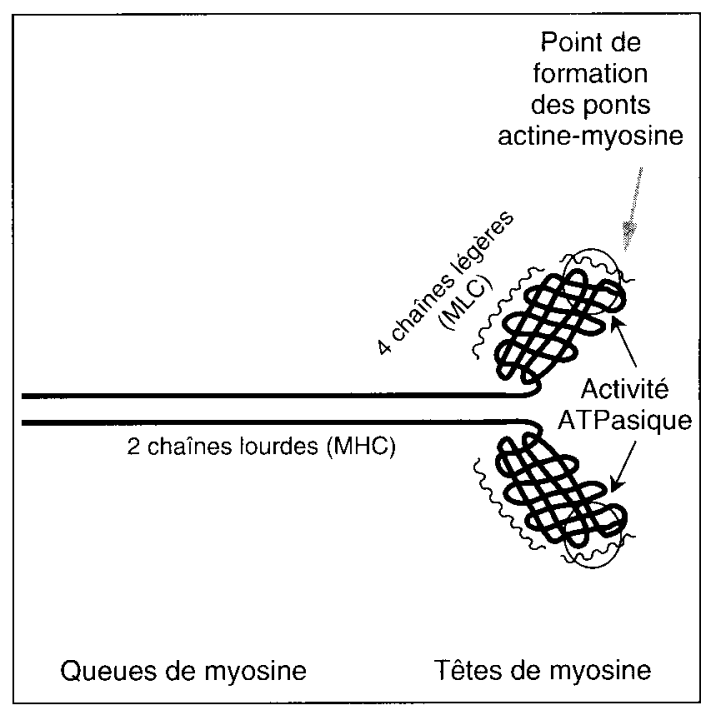

comme le muscle de la joue des mammifères herbivores (masséter) qui sert à la mastication. Cette proportion élevée en fibres lentes est directement en rapport avec sa fonction puisqu'il doit se contracter pendant plusieurs heures pour permettre au cheval de mécher le fourrage. C'est le type même de travail musculaire d'endurance.

Les fibres rapides (II) sont capables de délivrer des contractions puissantes pendant une durée plus limitée. Le cheval est une espèce dont les muscles locomoteurs sont très riches en fibres rapides : environ $80 \%$ (IIA+IIB) contre $40 \%$ chez l'homme pour le muscle fessier moyen.

Les fibres II A sont à la fois rapides et dotées d'un métabolisme énergétique aérobie utilisant principalement le glycogène comme source d'énergie par l'intermédiaire de la glycolyse aérobie et du cycle de Krebs. Elles possèdent pratiquement autant de mitochondries, de myoglobine et de capillaires que les fibres lentes. Ce sont donc des fibres rapides adaptées à soutenir un exercice musculaire de résistance : des contractions de forte puissance, développées pendant quelques minutes. 
Les fibres IIB sont encore plus puissantes que les IIA. La puissance élevée des contractions est associée à une fourniture rapide d'ATP mais limitée dans le temps. Elles consomment de la créatine phosphate et du glycogène par la voie de la glycolyse anaérobie qui aboutit à la production d'acide lactique. Leurs caractéristiques en font des fibres spécialisées pour fournir des contractions très puissantes durant quelques dizaines de secondes. Peu riches en myoglobine, elles apparaissent rouge clair et présentent une grande surface (sur les coupes transversales) qui est en rapport avec leur grande puissance de contraction.

Les fibres II C ont des propriétés intermédiaires entre les fibres lentes I et les fibres rapides IIA. Elles sont présentes en très faible proportion chez l'adulte comme chez le poulain. Dans le cas de nécrose musculaire faisant suite à une myosite, leur nombre augmente comme si elles étaient un stade de maturation obligatoire entre la cellule indifférenciée (cellule satellite) et la fibre musculaire spécialisée lente (I) ou rapide (IIA et IIB). Il s'agirait donc d'une forme transitoire de fibre possédant à la fois des chaînes de myosine rapide et lente.

\section{2 / Relation entre la fonction et la typologie d'un muscle}

La typologie d'un muscle dépend avant tout de sa fonction principale : posturale, propulsive, respiratoire ou autre. La figure 4 présente une synthèse des résultats d'analyse de différents muscles du cheval (Gunn 1978, Snow et Guy 1980, Barrey et al 1994, Valette et al 1994). Le muscle de la joue (masséter) est constitué à $100 \%$ de fibres lentes tandis que le muscle cutané du grasset (cutaneus tronci) est constitué à $98,5 \%$ de fibres rapides. Le premier actionne la méchoire inférieure pour la mastication des aliments et le second agite vivement le pli du grasset pour chasser les insectes. Le diaphragme est riche en fibres lentes (près de $70 \%$ ) qui permettent d'assurer la fonction ventilatoire en principe sans fatigue. Les muscles locomoteurs ont des compositions intermédiaires qui sont liées à leurs fonctions motrices prédominantes : posturale (antigravitaire) ou propulsive. Parmi ceux-ci, les muscles propulseurs de l'arrière-main comme le semi-tendineux (semitendinosus), le vaste latéral de la cuisse (vastus lateralis) et le fessier moyen (gluteus medius) sont les plus riches en fibres rapides. Chez d'autres mammifères (chien, wallabie), les muscles situés en profondeur à proximité des rayons osseux des membres sont plus riches en fibres lentes que les autres muscles placés plus superficiellement (Amstrong 1981).

\section{3 / Hétérogénéité de la typologie intra-musculaire}

Plusieurs études ont montré qu'il existait une répartition non homogène des types de fibres à l'intérieur de chaque muscle. A titre d'exemple, le fessier moyen (gluteus medius) comporte davantage de fibres lentes en profondeur qu'en surface (Kai 1984, Bruce et Turek 1985). A l'inverse, les fibres rapides IIB sont présentes en plus grande proportion en surface tandis que les fibres IIA ont une répartition plus homogène. Les muscles semi-tendineux (semitendinosus) et pectoral transverse (pectoralis transversus) présentent davantage de fibres lentes en profondeur (Gunn 1978). Ces gradients de fibres à l'intérieur d'un même

Figure 4. Proportion de fibres lentes dans différents muscles chez le cheval (d'après Snow et Guy 1980, Barrey et al 1994, Valette et al 1994).

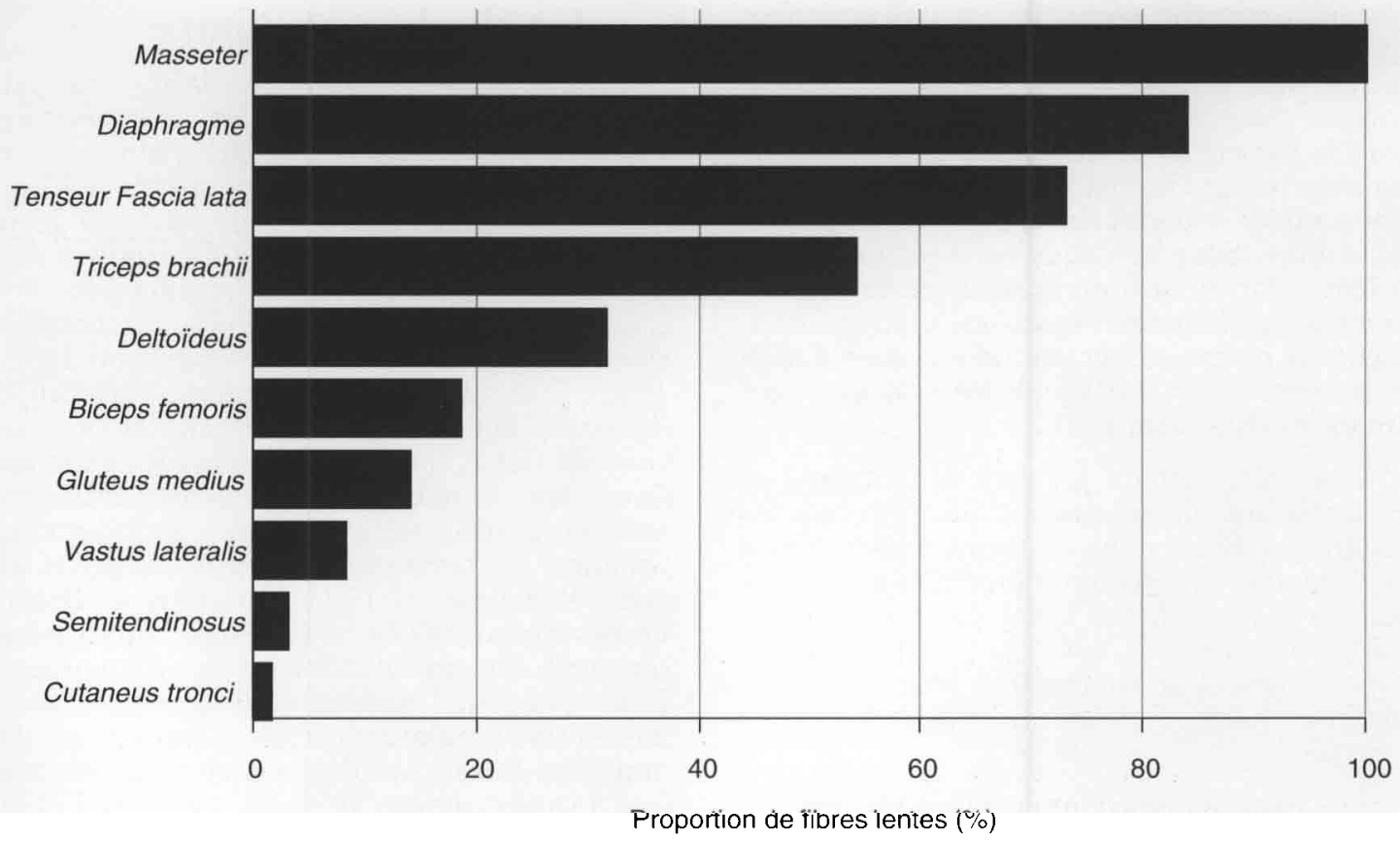

Chaque muscle contient en général plusieurs types de fibres, en proportions variables selon sa fonction. 
muscle suggèrent que les différentes parties du muscle ne jouent pas exactement le même rôle. Pour le semitendineux, la partie la plus profonde (riche en fibres lentes) assure le tonus musculaire nécessaire à la fonction posturale, tandis que la partie la plus superficielle (riche en fibres rapides) intervient dans la fonction de propulsion pour pousser le membre postérieur vers l'arrière (extension fémorale et flexion fémoro-tibiale).

Chez le rat, les muscles extenseurs profonds sont riches en fibres lentes et homogènes (soleus), tandis que les extenseurs placés plus superficiellement présentent un gradient croissant de fibres lentes de l'extérieur vers l'intérieur (plantaris, gastrocnemius) (Amstrong 1981).

Certains muscles présentent de véritables compartiments ayant des proportions en fibres assez distinctes. Le fessier moyen, par exemple, est constitué de deux compartiments séparés par une membrane de collagène : l'un dorsal est plus riche en fibres lentes ( 25 contre $11 \%$ dans le ventral), l'autre ventral est plus riche en fibres rapides $(89 \%$ contre $75 \%$ dans le dorsal) (Bruce et al 1993).

Cette variation de composition en fibres à l'intérieur de chaque muscle doit être prise en compte pour interpréter les résultats d'analyse provenant d'échantillons musculaires. Elle pose des difficultés de reproductibilité des biopsies et plus particulièrement des microprélèvements. En standardisant l'emplacement et la profondeur de la biopsie afin de minimiser la source de variation intra-musculaire, les résultats de deux biopsies successives varient tout de même de 6 à $12 \%$ selon le type de fibre (Lopez-Rivero et al 1991).

\section{4 / Influence de l'innervation et notion d'unité motrice}

Les fibres musculaires sont commandées par des motoneurones dont le corps cellulaire est situé dans la moelle épinière. Un nerf moteur comprend de nombreux motoneurones qui innervent chacun plusieurs fibres musculaires. Chaque fibre reçoit une seule terminaison nerveuse appelée synapse. Cette interface avec le muscle ou plaque motrice est le lieu de passage et de traduction de l'influx nerveux qui contrôle le déclenchement des contractions de la fibre. Sous la plaque motrice, on observe à l'intérieur de la fibre musculaire un regroupement de plusieurs noyaux cellulaires fondamentaux et une concentration marquée d'ARN messagers à partir desquels les protéines sont traduites (Klarsfeld 1991).

Une unité motrice est donc constituée d'un motoneurone, de ses axones qui innervent les différentes fibres dans un même muscle. Cette organisation arborescente implique que toutes les fibres musculaires innervées par le même motoneurone ont les mêmes propriétés contractiles et se contractent simultanément lorsque l'influx nerveux se propage jusqu'aux synapses.

Les expériences d'innervation croisée ont montré que les caractéristiques d'activité élec- trique du motoneurone déterminaient la typologie de la fibre musculaire innervée (Buller et al 1960, Monod et Flandrois 1985). Les motoneurones à conduction lente et seuil d'activation bas innervent les fibres lentes. Ces dernières sont donc les premières à être activées lorsqu'un mouvement est initié. Les unités motrices sont peu fatigables et peuvent ainsi se contracter un grand nombre de fois sans que la force de contraction diminue. A l'opposé les fibres rapides sont innervées par des motoneurones à conduction rapide et au seuil d'activation plus haut. Les unités motrices rapides sont stimulées après les fibres lentes lorsque l'effort demandé s'intensifie. Les unités motrices rapides sont constituées d'un plus grand nombre de fibres que les unités motrices lentes. Cette organisation est donc particulièrement adaptée aux contractions brèves de forte puissance. Les unités motrices des fibres IIA sont moins fatigables que celles des IIB mais elles sont aussi moins puissantes.

En fonction de la puissance de la contraction musculaire, on assiste au recrutement d'un nombre croissant d'unités motrices pour développer les forces nécessaires à la réalisation du mouvement. De plus, il existe une hiérarchie de recrutement parmi les unités motrices en fonction de la puissance de la contraction : les unités motrices lentes entrent en action les premières, puis interviennent les unités rapides. Selon la durée et la puissance de l'exercice, la proportion de chaque type des fibres change. Plus on demande de la puissance sur une durée brève ( 1 minute), plus les fibres anaérobies rapides (IIB) interviennent en grande proportion. A l'inverse, si l'effort est prolongé plusieurs minutes, voire plusieurs heures, les contractions de puissance plus modérée ne peuvent être assurées que par les fibres aérobies (I et IIA).

\section{3 / Facteurs de variation de la typologie musculaire}

Comme toutes les protéines, la myosine est synthétisée d'après l'information génétique apportée par les ARN messagers, eux-mêmes provenant de la transcription de l'ADN nucléaire. La séquence des acides aminés de la myosine est donc parfaitement déterminée par le code génétique et les gènes codant pour les chaînes de la myosine et l'actine sont identifiés chez la souris et l'homme (Buckingham 1989, 1992). Il semble d'ailleurs que la variabilité inter-spécifique des chaînes lourdes de la myosine soit faible, tout du moins chez les mammifêres, dans la mesure où les mêmes anticorps anti-myosine lente croisent sur le rat, l'homme, les bovins et les équins (Léger et al 1985, Robelin et al 1993, Valette et al 1994). Le déterminisme de la typologie musculaire comporte donc inévitablement une composante génétique mais l'expression des gènes est soumise à une régulation complexe qui intègre de multiples facteurs endogènes et exogènes tels que l'innervation, le sexe, l'âge et l'état d'entraînement (Periasamy et al 1989). 
Figure 5. Variation inter-races de la typologie musculaire du muscle fessier moyen chez le cheval (d'après Snow et Guy 1980).

Proportion de fibres (\%)

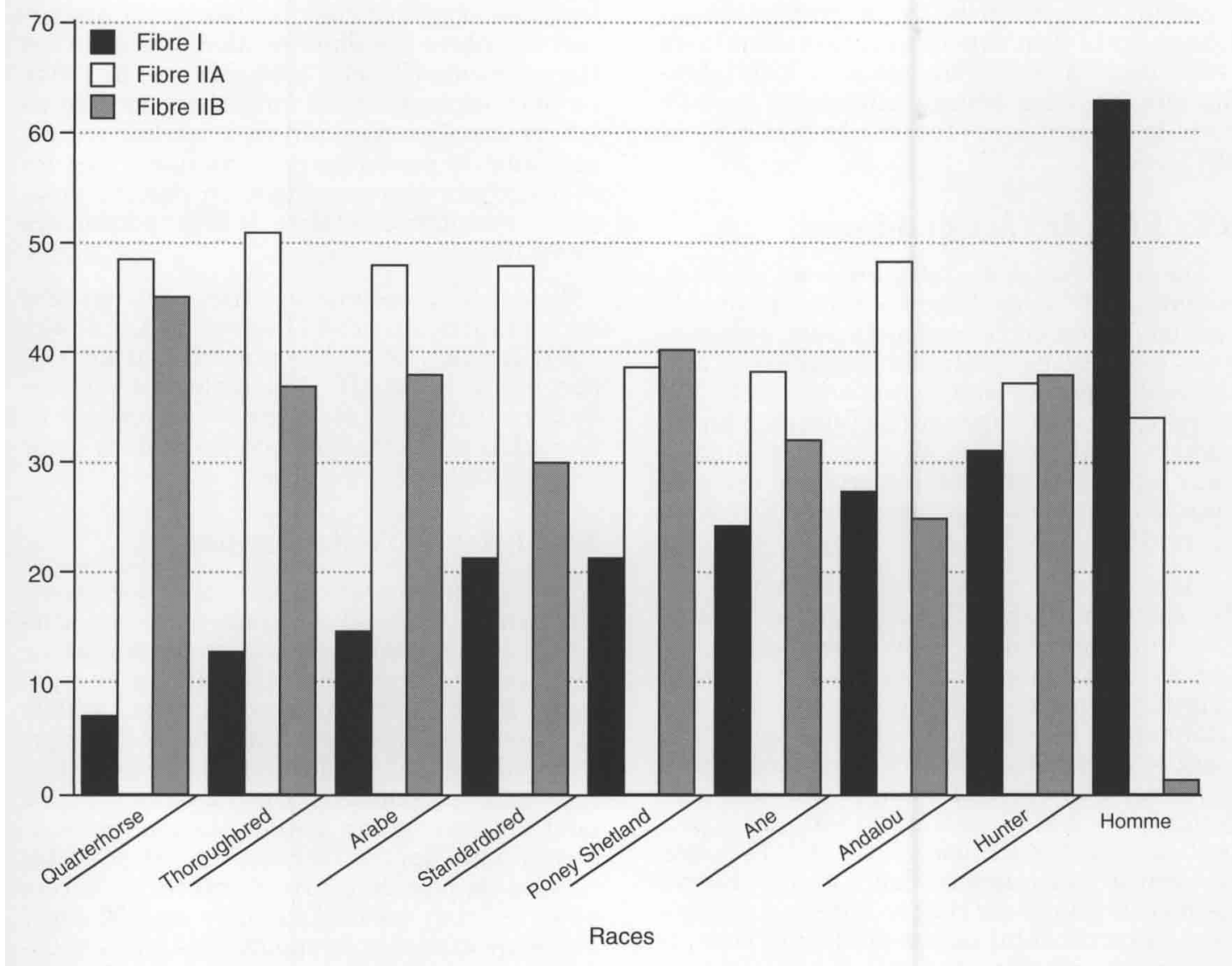

A l'intérieur d'une fibre (longue de plusieurs dizaines de $\mathrm{cm}$ ) qui comprend des centaines de noyaux cellulaires, il semble que l'expression des gènes soit dépendante de la répartition spatiale des noyaux dans la fibre (Klarsfeld 1991). Ainsi, les noyaux siués sous la plaque motrice auraient un rôle régulateur prépondérant par rapport aux noyaux secondaires placés le long de la fibre.

\section{1 / Facteurs génétiques}

Chez l'homme, plusieurs études ont montré une plus grande ressemblance de la typologie musculaire entre des vrais jumeaux (homozygotes) qu'entre des faux jumeaux (hétérozygotes) ou frères et soeurs (Komi et al 1973, 1977, Prudhomme et al 1984, Simoneau et al 1986). Les facteurs génétiques ont surtout une influence sur la proportion de fibres lentes/rapides alors que la proportion de fibres rapides IIA/IIB est davantage soumise à des facteurs extra-génétiques tels que l'âge, le sexe et l'entraînement. Chez le cheval, aucune étude n'a mis en évidence la composante génétique de ces caractéristiques musculaires du fait de la lourdeur des analyses histologiques à mettre en ceuvre pour aborder une telle étude de génétique quantitative. Par contre, l'influence de la race sur la typologie moyenne de certains muscles locomoteurs a été présentée à plusieurs reprises (figure 5) (Snow et Guy 1980). Pour un muscle locomoteur majeur comme le fessier moyen, les races de course telles que le Pur sang (Thoroughbred) sont mieux dotées en fibres rapides. A l'inverse, les races plus rustiques (Hunter, Andalou) possèdent plus de fibres lentes, moins puissantes mais aussi moins fatigables. Une étude portant sur la typologie des muscles semitendineux, pectoral transverse et diaphragme de chevaux appartenant à trois groupes génétiques (Pur sang, croisés de Pur sang et races de selle rustiques) montre des différences significatives entre groupes pour ces deux muscles. La proportion de fibres rapides décroît des chevaux Pur sang au groupe des races rustiques, les croisés de Pur sang se situant entre les deux (Gunn 1978). Par ailleurs, l'étude du nombre total de fibres d'une section transversale du semitendineux dans ces mêmes groupes montre que les Pur sang ont un nombre total de fibres plus élevé (Gunn 1979).

Pour l'instant, il n'existe pas d'estimation de l'héritabilité des caractères musculaires chez le cheval mais quelques valeurs sont proposées dans d'autres espèces de mammifères dont les processus de myogenèse et de

\section{Les muscles \\ locomoteurs contiennent plus de fibres rapides chez les chevaux de course par rapport aux races plus rustiques.}


régulation sont très proches (Barrey 1994). Chez l'athlète humain, la proportion en fibres lentes du muscle vaste latéral a une héritabilité de 30 à $55 \%$ (Lortie et al 1984). Chez le mouton, l'héritabilité de la proportion en fibres lentes du muscle scuto-auriculaire serait de 27 à $46 \%$ (Vigneron et al 1986), tandis que, chez les bovins, elle serait de $24 \%$ pour le muscle long dorsal (Andersen et al 1977).

\section{2 / Effet de l'âge et du sexe}

Du stade foetal de 180 j jusqu'au stade de poulain de 18 mois, la composition du muscle semitendineux en fibres lentes augmente progressivement de quelques pourcents à plusieurs dizaines de pourcents (Gunn 1991). Par ailleurs, entre la naissance et l'âge de 2 ans le nombre total de fibres du muscle semitendineux augmente et cette hyperplasie est plus marquée chez les chevaux de race Pur sang (Gunn 1979).

Deux études, réalisées respectivement sur des chevaux de race Trotteurs américains et Pur sang, ont montré que la proportion en fibres lentes du muscle fessier moyen augmentait légèrement de l'âge de 1 an à 4 ans (Roneus et Lindholm 1991, Roneus 1993). Chez le Trotteur américain, la proportion en fibres rapides IIA tend à augmenter de 8 à 10 points entre 1 et 6 ans d'âge tandis que la proportion en fibres IIB décroît de 13 à 14 points pendant le même temps (figure 6). On observe les mêmes tendances chez le Pur sang. Le protocole expérimental de ces études ne permettait malheureusement pas de distinguer l'influence de l'âge et celle de l'accumulation

Figure 6. Evolution de la typologie musculaire du muscle fessier moyen (gluteus medius) en fonction de l'âge du cheval Trotteur américain (d'après Roneus 1993).

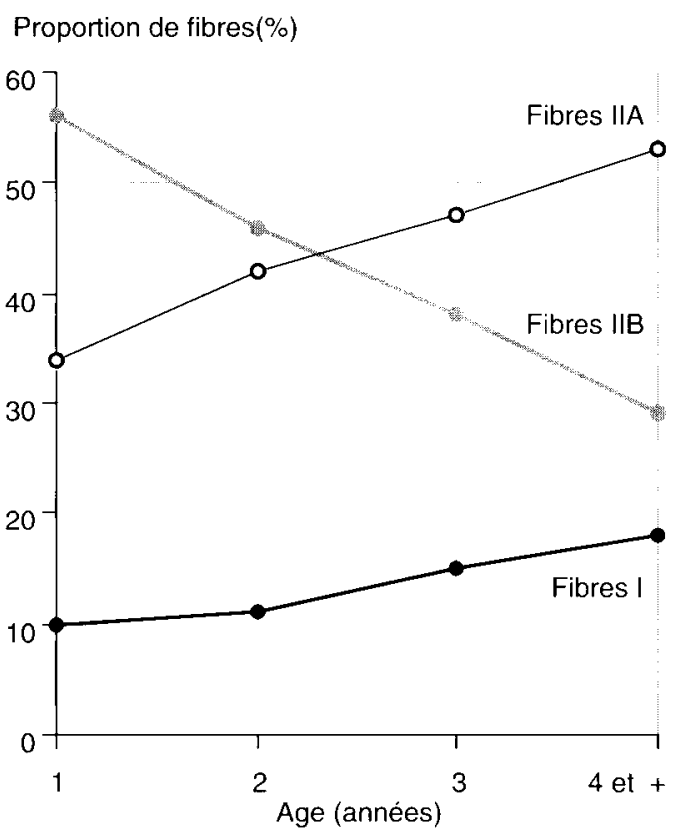

du travail d'entraînement qui commence vers 18 mois chez les chevaux de course. Chez les bovins, l'évolution des proportions en fibres rapides évolue avec l'âge en sens inverse de la tendance observée chez le cheval : la proportion des fibres IIA diminue alors que celle des IIB augmente (Robelin 1990, Micol et al 1993). Ce phénomène pourrait être lié au manque ou à l'absence d'exercice physique réalisé par ces animaux de producteurs de viande. Chez les chevaux mis au repos forcé, on observe aussi une augmentation relative de la proportion des fibres IIB.

Seules les proportions en fibres rapides sont dépendantes du sexe (Roneus et Lindholm 1991, Roneus 1993). Les juments ont un rapport en fibres IIA/IIB plus faible, des surfaces de fibres IIA moins étendues et des surfaces de fibres IIB plus étendues que les étalons (pour le muscle fessier moyen).

\section{3 / Effet de l'entraînement}

De nombreux travaux ont mis en évidence l'influence du travail d'entraînement sur l'évolution de la typologie musculaire et des activités enzymatiques du métabolisme énergétique. Il est en effet primordial pour l'athlète et l'entraîneur de savoir si un travail physique particulier peut modifier favorablement la typologie musculaire. En d'autres termes, peut-on développer les adaptations musculaires pour spécialiser un cheval pour les courses de plat de courte distance, ou encore pour les courses d'endurance de $150 \mathrm{~km}$ ? Plusieurs études ont rapporté des effets significatifs du travail d'entraînement sur la composition en fibres musculaires de certains muscles. La plupart des travaux ont porté sur les chevaux de courses au trot et au galop. Quelques données sont également disponibles sur le cheval d'endurance. D'une manière générale, les changements de la proportion des fibres musculaires dépendent de la nature, de l'intensité, de la durée cumulée du travail d'entraînement et de l'âge du sujet entraîné.

Chez les chevaux de courses trotteurs adultes, on observe des modifications de la proportion et des surfaces de fibres du muscle fessier moyen après une période d'entraînement intense de deux semaines (Gottlieb-Vedi 1988). La proportion en fibres IIA augmente tandis que celle en fibres IIB diminue et que celle en fibre I reste inchangée. Après 12 semaines d'entraînement, la composition en fibres reste semblable à celle observée après les deux premières semaines. La surface relative des fibres IIA s'accroît également pendant les deux premières semaines puis se stabilise. Chez le galopeur les mêmes tendances sont observées après une période d'entraînement intense. Le travail en résistance (puissance maximale pendant plus de 5 minutes) augmente donc le rapport des fibres IIA/IIB et la surface relative des fibres IIA.

Chez les chevaux Pur sang, âgés de 2 à 9 ans, une période d'entraînement intense de 6 semaines provoque une élévation de $28 \%$ du 
pourcentage de IIA et une baisse de $29 \%$ des IIB. Six semaines après l'arrêt de l'entraînement, la composition en fibres reste inchangée (Sinha et al 1991). D'autres travaux montrent une plus grande réversibilité de l'adaptation musculaire consécutive à l'entraînement. Chez un athlète initialement entraîné, l'immobilisation forcée d'un muscle locomoteur majeur pendant 5 semaines induit une réduction du pourcentage de fibres I et une augmentation apparente du pourcentage de fibres IIB et cette tendance est également observée chez le cheval (Häggmark et al 1981).

Un travail d'entraînement conventionnel de 6 semaines, moins intense que le précédent, augmente la surface de fibres rapides mais ne modifie pas les proportions (Foreman et al 1990).

Chez les chevaux Arabes et Andalous non entraînés, modérément entraînés ou très entraînés pour la course d'endurance (très longue distance à faible vitesse), on constate aussi un effet significatif de l'entraînement sur la composition en fibres rapides du muscle fessier moyen. Le travail en endurance pratiqué sur une période de 6 mois augmente donc le rapport IIA/IIB chez les chevaux les plus entraînés (Lopez-Rivero et al 1991).

Chez le poney, un travail d'entraînement en résistance d'une durée de trois mois induit des modifications de la typologie du muscle Triceps brachial (Valette et al 1990). A la fin de la période d'entraînement, le pourcentage en fibres lentes I a augmenté de 13 points. Le rapport des fibres rapides IIA/IIB est passé de 1,5 à 3,5 avec une persistance du pourcentage des fibres IIA $(40 \%)$ et une diminution de celui des IIB (27 à 12\%).

Dans tous les cas, l'entraînement suffisamment intense accroît la proportion de fibres rapides aérobies IIA au détriment des fibres rapides anaérobies IIB. Parallèlement, la surface relative des fibres IIA augmente.

Sous l'influence d'un entraînement intense en résistance, on observe en général une hypertrophie musculaire qui s'explique par la conjonction de deux phénomènes : l'augmentation de surface de certaines fibres et l'accroissement du nombre total de fibres. Cette hyperplasie a été démontrée chez le chat et le rat (Mikesky et al 1991, Tamaki et al 1992).

Toutes ces modifications structurales s'accompagnent d'améliorations de l'activité enzymatique des voies métaboliques aérobies, le tout contribuant à augmenter la puissance musculaire tout en réduisant la fatigabilité.

La composition en fibres musculaires change au cours du temps sous l'influence d'un programme d'entraînement, et plusieurs mécanismes expliquent ces modifications apparentes de la proportion des différentes fibres. La pratique de l'électromyostimulation est utilisée pour créer des modèles d'étude de la transformation des fibres sous l'influence d'un entraînement physique programmé (Bigard et al 1991). L'application d'un courant électrique de basse fréquence ( $10 \mathrm{~Hz}$ ) sur un muscle normalement innervé reproduit artificiellement la stimulation physiologique du motoneurone lent. Ces courants induisent in vivo des modifications progressives de la proportion des fibres avec des transformations apparentes dans le sens suivant : IIB->IIA->IIC->I (Pette et al 1976). Par contre, l'application de courant haute fréquence $(40$ à $60 \mathrm{~Hz}$ ) sur le muscle normalement innervé ne transforme pas les fibres lentes en rapides.

L'étude des modifications cellulaires et moléculaires ont mis en évidence les deux mécanismes responsables du changement de la typologie : l'élimination suivie du remplacement intégral des fibres ou la transformation moléculaire de la myosine des fibres en place (travaux cités par Bigard et al 1991). Il semble que ce dernier processus soit le principal responsable du remodelage musculaire. Les transformations moléculaires se font par un changement d'assemblages des sous-unités de la myosine (chaînes légères et chaînes lourdes). Après une période prolongée d'électrostimulation d'un muscle rapide de lapin, l'expression des chaînes légères rapides diminue au profit d'une augmentation de la synthèse des chaînes légères lentes. On constate, parallèlement, une baisse de l'expression des chaînes lourdes rapides IIB, suivie d'une élévation des chaînes lourdes rapides IIA, et finalement, après quelques semaines d'expérimentation, une apparition massive de chaînes lourdes lentes I.

Le processus de remplacement intégral des fibres s'opère par une destruction sélective des fibres IIB qui sont peu à peu remplacées par des nouvelles fibres de type IIC. Après quelques jours d'électrostimulation d'un muscle de lapin, on constate que certaines fibres IIB sont dégénérescentes et que la population des fibres IIC initialement très faible augmente sensiblement. Les fibres naissantes IIC évoluent ensuite vers un type I ou IIA.

Les modifications de typologie musculaire sous l'influence de l'entraînement ou d'électrostimulation basse fréquence semblent toujours se faire dans le même sens. Ainsi, le muscle s'adapte pour fournir un travail en résistance tout en étant moins fatigable. A l'opposé, il semble impossible, dans les conditions physiologiques normales, de gagner de la puissance au détriment de la fatigabilité en provoquant des transformations de fibres lentes en fibres rapides. La proportion de fibres rapides (II/I) n'est donc pas ou très peu influençable par l'entraînement, ce qui laisse penser que l'aptitude musculaire à fournir un effort de grande puissance dépend davantage de l'origine génétique que l'aptitude à fournir un effort d'endurance (Howald 1982). Ces données de physiologie musculaire justifieraient la prédominance de la génétique dans le choix des chevaux de courses spécialisés sur les courtes distances ("flyer").
L'entraînement intense modifie la typologie musculaire, surtout le rapport entre les fibres rapides IIA et IIB. 


\section{4 / Relation entre la typologie musculaire et la performance physique}

Chez l'athlète humain plusieurs études ont montré les rapports entre la typologie musculaire et la discipline sportive pratiquée (Bergh et al 1978) (figure 7). La relation entre la typologie du vaste latéral, extenseur du genou, et la performance de contraction a été démontrée chez l'homme par une série de tests d'extension du genou (Thorstensson et al 1976). Il existe une corrélation modérée $(0,50)$ de la puissance maximale avec le pourcentage de fibres rapides. Il en est de même pour la vitesse maximale de contraction.

ll existe également une relation entre la consommation d'oxygène $\left(\mathrm{VO}_{2}\right)$ et la typologie musculaire des muscles locomoteurs : le pourcentage de fibres lentes est plus élevé dans les disciplines nécessitant une proportion plus élevée de la consommation maximale d'oxygène $\left(\mathrm{VO}_{2} \max \right)$ (Bergh 1978). Les sprinters ont plus de $75 \%$ de fibres rapides, les sauteurs plus de $50 \%$. A l'opposé, les skieurs de fond et les marathoniens ont de $60 \%$ à $75 \%$ de fibres lentes (Saltin et al 1977). Ces compositions en fibres sont le résultat d'une spécialisation des athlètes, à la fois par la sélection sportive des individus les plus aptes et par la pratique d'un entraînement spécifique.

Figure 7. Pourcentage de fibres lentes (I) en fonction de la discipline sportive pratiquée par des athlètes humains (d'après Bergh et al 1978).

Proportion de fibres lentes (\%)

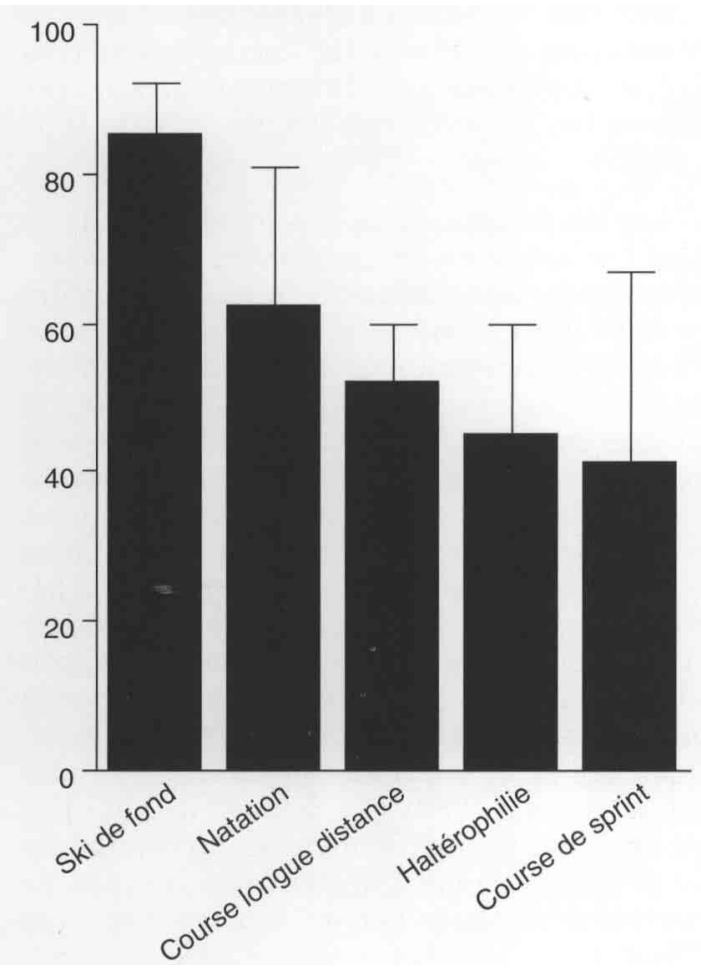

\section{1 / Typologie musculaire et aptitude du cheval à l'effort de puissance}

Chez le cheval, certaines races ont été sélectionnées d'après leurs performances en course ce qui explique, par exemple, la spécificité de la typologie musculaire du Pur sang, du Quarterhorse ou du Trotteur. Ces races ont plus de $80 \%$ de fibres rapides dans le muscle fessier moyen.

Quelques données chez le Pur sang indiquent que les sprinters courts ont davantage de fibres IIB tandis que les sprinters longs ont davantage de IIA (McMiken 1986). Les meilleurs chevaux ont des fibres de surface plus homogènes et les densités capillaire et mitochondriale sont plus élevées. Les meilleurs chevaux de courses Quarterhorses ont un plus grand pourcentage de fibres IIB, ce sont des purs sprinters. Une étude sur le cheval Trotteur américain a montré que sa vitesse sous-maximale V200 (vitesse induisant 200 battements cardiaques par minute) mesurée au cours d'un test d'effort, était corrélée positivement avec le pourcentage de fibres rapides IIA et négativement avec celui des fibres rapides IIB (Valberg et al 1985).

La typologie moyenne du cheval de concours hippique est encore inconnue mais il est probable qu'à haut niveau les meilleurs sauteurs aient une forte proportion de fibres rapides dans les muscles de l'avant-main et de l'arrière-main, impliqués dans les mouvements du saut.

\section{2 / Typologie musculaire et aptitude à l'effort prolongé}

Les chevaux spécialisés dans la discipline des courses d'endurance ont des muscles fessiers qui comportent environ $30 \%$ de fibres lentes et 40 à $50 \%$ de fibres IIA (Lopez-Rivero et al 1991). Chez les poneys ayant eu un entraînement de 3 mois à leur vitesse seuil VLa4 (vitesse induisant une lactatémie de 4 $\mathrm{mmol} / \mathrm{l}$ ), il existe une corrélation positive entre la composition en fibres lentes I et la vitesse au seuil anaérobie (Valette et al 1990). D'après ces résultats, l'adaptation musculaire à l'effort d'endurance dépendrait de la composition en fibres aérobies de type I et IIA.

\section{Conclusion}

\section{Peut-on prédire la performance sportive d'après la typologie musculaire?}

Chez le cheval comme chez l'athlète humain, la performance sportive dans une discipline donnée résulte d'un ensemble de facteurs favorables intrinsèques et extrinsèques à l'animal. Les qualités neuro-sensorielles, la capacité cardio-respiratoire, les propriétés contractiles des muscles et les caractéristiques locomotrices de l'individu sont les principaux facteurs intrinsèques impliqués dans l'effort physique. La performance sportive est donc un 
caractère très composite qui est quasiment imprévisible du fait du grand nombre de facteurs à prendre en compte. Mis à part les facteurs environnementaux, la performance physique est semble-t-il modérément corrélée à la composition en fibres des muscles qui y contribuent. Les éleveurs de chevaux de course l'ont déjà constaté empiriquement depuis longtemps en sélectionnant indirectement les caractères musculaires contractiles au travers des performances en course. L'étude du déterminisme de la typologie musculaire a montré le rôle du système nerveux dans la différenciation des fibres musculaires. Les propriétés de ce système régulateur de toutes les fonctions organiques transparaissent au travers des caractéristiques musculaires, cardiaques et comportementales du cheval. Sa réactivité pendant l'effort et son tempérament ressentis par l'utilisateur ne sont donc pas sans rapport avec ses caractéristiques musculaires et la notion empirique de "cheval de sang" correspond bien à des qualités musculaires particulières.

La puissance musculaire qui dépend $\mathrm{du}$ nombre total de fibres, de leurs propriétés contractiles et énergétiques, n'est qu'un des éléments de la production de l'effort physique. Quelle est son importance relative dans chacune des disciplines hippiques? Dans les courses rapides, la typologie musculaire a semble-t-il un rôle prédominant et le génotype du cheval aurait d'autant plus d'importance que la course se joue sur une courte distance. Pour les courses d'endurance, la typologie musculaire favorable est probablement un facteur nécessaire mais non limitant dans la mesure où le travail d'entraînement peut l'améliorer. Pour le cheval de concours hippique, on manque encore de données mais, à haut niveau, on peut supposer que la puissance musculaire maximale disponible au moment de l'appel départage des chevaux techniquement semblables.

\section{Pourra-t-on utiliser la typologie musculaire pour la sélection?}

Pour envisager une telle application chez le cheval, il faudrait d'une part démontrer que certaines typologies musculaires sont favorables à la performance sportive dans les diverses disciplines hippiques et, d'autre part, estimer l'héritabilité de la typologie des principaux muscles impliqués dans l'effort. Chez l'athlète humain, la typologie musculaire a sans aucun doute des rapports étroits avec la performance sportive dans les disciplines où la composante physique est dominante (sprint, demi-fond, marathon, saut, ski de fond). Dans le cas du cheval, cela reste encore à démontrer mais l'existence de races spécialisées pour la course, bien dotées en fibres rapides apporte déjà un élément de réponse. La connaissance des principaux facteurs de variation de la typologie musculaire chez le cheval montre l'importance de l'âge et de l'entraînement mais aussi de la génétique. Les effets de la race du cheval et les héritabilités moyennes à fortes obtenues chez les autres espèces de mammifères sont des arguments en faveur d'une influence significative de l'origine génétique sur la composition en fibres rapides et lentes des muscles locomoteurs chez le cheval. Avec le développement des nouvelles techniques immunologiques du dosage des chaînes lourdes de la myosine, il est maintenant possible d'envisager des études sur des effectifs plus importants que ne le permettaient les techniques antérieures d'histoenzymologie. Les applications génétiques deviennent donc envisageables.

\section{Références bibliographiques}

Amstrong R.B., 1981. Recruitment of muscle and fibres within muscles in running animals. Symp. zool. Soc. Lond. 48, 289-304.

Andersen B.B., Lykke T., Kousgaard K., Buchter L., Wismer Pedersen J., 1977. Growth, feed utilization, carcass quality and meat quality in danish dualpurpose cattle. Beretning fra Statens Husdyrbrugs forsog, 453, 60-68.

Barrey E., 1994. L'analyse de la typologie musculaire peut-elle constituer un critère de sélection? CEREOPA, 2 mars 1994, Paris.

Barrey E., Valette J.P., Picard B., Geay Y., 1994. Determination of the slow myosin heavy chain content in equine gluteus medius by an ELISA method. Equine Vet. J., soumis pour publication.

Bergh U., 1978. Maximal oxygen uptake and muscle fiber types in trained and untrained humans. Med. Sci. Sports, 10, 151.
Bergstrom J., 1975. Percutaneous needle biopsy of skeletal muscle in physiological and clinical research. Scand. J. Clin. Lab. Invest., 35, 609-616.

Bigard A.X., Canon F., Guezennec C.Y., 1991. Conséquences histologiques et métaboliques de l'électrostimulation - revue de la littérature. Science et Sport, 6, 275-292.

Billeter R., Lador J., Howald H., Straub R., 1987. Gel electrophoresis of proteins from single equine muscle fibers. in Gillespie J.R., Robinson N.E. (Eds) : Equine Exercise Physiology, 2, 359-366.

Brooke M.M., Kaiser K.K., 1970. Muscle fiber types : how many and what kind? Arch. Neurol., 23, 369379 .

Bruce V.L., Turek R.J., 1985. Muscle fibre variation in the gluteus medius of the horse. Equine Vet. J., 17, 317-321. 
Bruce V.L., Turek R.J., Schrung W.A., 1993. Muscle fibre compartmentalisation in the gluteus medius of the horse. Equine Vet. J., 25, 69-72.

Buckingham M.E., 1989. The control of muscle gene expression : A review of molecular studies on the production and processing of primary transcripts. British Medical Bulletin, 45(3), 608-629.

Buckingham M.E., 1992. Making muscles in mammals. Trends in Genetics, 8(4), 144-149

Buller A.J., Eccles J.C., Eccles R.M., 1960. Interactions between motoneurones and muscles in respect of the characteristic speeds of their responses. J. Physiol., 150, 417-439.

Danieli Betto D., Zerbato E., Betto R., 1986. Type 1, $2 \mathrm{~A}$, and $2 \mathrm{~B}$ myosin heavy chain electrophoretic analysis of rat muscle fibers. Biochem. Biophys. Res. Communic., 138(2), 981-987.

Foreman H.J., Warwick M. B., Allen J. R., Matoba H., Grant B.D., Gollnick P.D., 1990. Muscle responses of Thoroughbreds to conventional race training and detraining. Am. J. Vet. Res., 51, 909913

Gollnick P.D., 1982. Relationship of strength and endurance with skeletal muscle structure and metabolic potential. Int. J. Sports Med., 3, 26-32.

Gottlieb-Vedi M., 1988. Circulatory and muscle metabolic responses to draught work of varying intensity and duration in Standardbred horses. Thesis, Faculty of veterinary medicine, Swedish University of Agricultural Sciences, Uppsala.

Gunn H.M., 1978. Differences in the histochemical properties of skeletal muscles of different breeds of horses and dogs. J.Anat., 127, 615-634

Gunn H.M., 1979. Total fibre number in cross sections of semitendinosus in athletic and nonathletic horses and dogs. J. Anat., 128, 821-828.

Gunn H.M., 1991. Growth changes in skeletal muscle histochemistry of Thoroughbreds and other horses. in Persson S.G.B., Lindholm A. and Jeffcott L.B.(Eds) : Equine Exercise Physiology, 3, 245-256.

Häggmark T., Jansson E., Eriksson E., 1981. Fiber type area and metabolic potential of the thigh muscle in man after knee surgery and immobilization. Int. J. Sports Med., 2, 12-17.

Hermanson J.W., Hegemann-Monachelli M.T., Daaod M.J., LaFranboise W.A., 1991. Correlation of myosin isoforms with anatomical divisions in equine musculus biceps brachii. Acta Anat., 141, 369-376.

Horak V., Draber P., Hanak J., Matolin S., 1991. Fibre composition and tubulin localization in muscle of Thoroughbred sprinters and stayers. in Persson S.G.B., Lindholm A. and Jeffcott L.B.(Eds) : Equine Exercise Physiology, 3, 262-268.

Howald H., 1982. Training-induced morphological and functional changes in skeletal muscle. Int. J. Sports Med., 3, 1-12.

Kai M., 1984. Distribution of fiber types in equine middle gluteal muscle. Bull. Equine Res. Inst., 21, 46-50.

Klarsfeld A., 1991. La compartimentation des fibres musculaires - les noyaux d'une fibre musculaire sont-ils tous égaux ? La Recherche 228 , vol. 22, 116118.
Komi P.V., Klissouras V., Karvinen E., 1973. Genetic variation in neuromuscular performance, Int. Z. angew. Physiol. 31, 289-304.

Komi P.V., Viitasalo J.H.T., Havu M., Thorstensson A., Sjödin B., Karlsson J., 1977. Skeletal muscle fibres and muscle enzyme activities in monozygous and dizygous twins of both sexes, Acta Physiol. Scand., 100, 385-392.

Léger J.O.C., Bouvagnet P., Pau B., Roncucci R., Léger J.J., 1985. Levels of ventricular myosin fragments in human sera after myocardial infarction, determined with monoclonal antibodies to myosin heavy chains. Eur. J. Cell. Biol., 15, 422-429.

Lindholm A., Piehl K., 1974. Fibre composition, enzyme activity and concentrations of metabolites and electrolytes in muscles of Standardbred horses. Acta Vet. Scand., 15, 287-309.

Lopez-Rivero J.L., Morales-Lopez J.L., Galisteo A.M., Aguera E., 1991. Muscle fibre type composition in untrained and endurance-trained Andalusian and Arab horses. Equine Vet. J., 23, 9193

Lortie G., Simoneau J.A., Boulay M.R., Bouchard C., 1984. Muscle fiber type composition and enzyme activities in brothers and monozygotic twins. in Malina R.M. and Bouchard C. (Eds): Sport and Human Genetics, Olympic Scientific Congress Proceedings vol 4, 147-153. Human Kinetics Publishers, Champaign, IL.

McMiken D., 1986. Muscle fiber types and horse performance. Equine practice, 8(3), 6-14

Micol D., Robelin J., Geay Y., 1993. Composition corporelle et caractéristiques biologiques des muscles chez les bovins en croissance et à l'engrais. INRA Prod. Anim., 6, 61-69

Mikesky A.E., Giddings C.J., Matthews W., Gonyea W.J., 1991. Change in muscle fiber size and composition in response to heavy-resistance. Med. Sci. Sports Exerc., 19, 266-274.

Monod H., Flandrois R., 1985. Constitution et propriétés des fibres musculaires striées squelettiques. in Physiologie du Sport, Masson, Paris, 55-72

Periasamy M., Gregory P., Martin B.J., Stirewalt W., 1989. Regulation of myosin heavy-chain gene expression during skeletal-muscle hypertrophy. Biochem., 257, 691-698.

Pette D., Schnez U., 1977. Coexistence of fast and slow type myosin light chains in single muscle fibres during transformation as induced by long term stimulation. FEBS Lett., 83, 128-130.

Pette D., Muller W., Leisner E., Vrbova G., 1976. Time dependent effects on contractile properties fibre population, myosin light chains and enzymes of energy metabolism in intermittently and continuously stimulated fast-twicht muscles of the rabbit. Pflugers Arch., 364, 103-112.

Picard B., Léger J.O.C., Robelin J., 1994 Quantitative determination of type $1 \mathrm{MHC}$ in bovine muscle with anti myosin monoclonal antibodies. Meat Sci. (sous presse)

Prud'homme D., Bouchard C., Leblanc C., Landry F., Fontaine E., 1984. Sensitivity of maximal aerobic power to training is genotype-dependent, Med. Sci. Sports Exerc., 16(5), 489-493. 
Robelin J., 1990. Différenciation, croissance et développement cellulaire du tissu musculaire. INRA Prod. Anim., 3(4), 253-263.

Robelin J., Picard B., Listrat A., Jurie C., Barboiron C., Pons F., Geay Y., 1993. Myosin transition in semitendinosus muscle during fetal development of cattle : immunocytochemical and electrophoretic analyses. Repr. Nutr. Dev., 33, 25-41.

Roneus M., 1993. Muscle characteristics in Standardbreds of different ages and sexes. Equine Vet. J., 25, 143-146.

Roneus M., Lindholm A., 1991. Muscle characteristics in Thoroughbreds of different ages and sexes. Equine Vet. J., 23, 207-210.

Saltin B., Henriksson J., Nygaard E., Andersen P., Jansson J., 1977. Fiber types and metabolic potentials of skeletal muscles in sedentary man and endurance runners. Ann. N.Y. Acad. Sci., 301, 3-29.

Simoneau J.A., Lortie G., Boulay M.R., Marcotte M., Thibault M.C., Bouchard C., 1986. Inheritance of human skeletal muscle and anaerobic capacity adaptation to high-intensity intermittent training, Int. J. Sports Med., 7(3), 167-171.

Sinha A.K., Rose R., 1992. Muscle fiber typing in the horse: current problems, future directions. in Proc. Ass. Equine Sports Medicin, Fallbrook CA, 7-11.

Sinha A.K., Ray S.P., Rose R., 1991. Effect of training intensity and detraining on adaptations in different skeletal muscles. in Persson S.G.B., Lindholm A. and Jeffcott L.B.(Eds) : Equine Exercise Physiology, 3, 223-230.

Snow D.H., Guy P.S., 1976. Percutaneous needle muscle biopsy in the horse. Equine Vet. J., 8, 150-155.
Snow D.H., Guy P.S., 1980. Muscle fibre type composition of a number of limb muscles in different types of horse. Res.Vet.Sci., 28, 137-144.

Tamaki T., Uchiyama S., Nakano S., 1992. A weightlifting exercise model for inducing hypertrophy in the hindlimb muscles of rats. Med. Sci. Sports Exerc., 24(8), 881-886.

Thorstensson A., Grimby G., Karlsson J., 1976. Force-velocity and fiber composition in human knee extensor muscles. J. Appl. Physiol., 40, 12-16.

Valberg S., Essen-Gustavsson B., Lindholm A., Persson S., 1985. Energy metabolism in relation to skeletal muscle fibre properties during treadmill exercise. Equine Vet. J., 17, 439-444.

Valette J.P., Wolter R., Zouambi B., 1990. Relations entre le type histo-enzymologique des fibres musculaires et les critères physiologiques de l'aptitude sportive chez le poney. Rec. Méd. Vét, 166, 765-769.

Valette J.P., Barrey E., Jouglin M., 1994. Determination of slow myosin heavy chain content in various equine muscles by an ELISA method. Proc. Int. Conf. Equine Exerc. Physiol., 4, 11-15th july, Brisbane, Australia.

Vigneron P., Prud'Hon M., Touraille C., Valin C., Bouix J., Bibé B., 1986. Croissance des agneaux types de fibres musculaires et qualité de la viande. Muscles indicateurs. Journées de Recherche Ovins Caprins 1986.

Winkelmann D.A., Lowey S., Press J.L., 1983. Monoclonal antibodies localize changes on myosin heavy chain isoenzymes during avian myogenesis. Cell, 34, 295-306.

\section{Summary}

Contractile properties of muscle fibres and exercise performance in borse - a review.

The purpose of this paper is to present the state of knowledge about the relationship between the muscle fibres types and the exercise ability in horse. The strength and contractile speed of a muscle is related to the total number of twitching fibres and their contractile properties. Skeletal muscles are the engine of the athlete and lot of studies have been made in exercise physiology to determine the properties and the adaptations of the muscle fibres in response to chronic exercise. The technics of investigation, the physiological characteristics of slow and fast twitch fibres and factors which may affect the contractile properties are presented, successively. It is concluded that immunoenzymatic method of muscle fibre typing may be apply to selection.

BARREY E., 1994. Propriétés contractiles des fibres musculaires et performance physique chez le cheval. INRA Prod. Anim., 7(1), 41-53. 